\section{Origen, evolución y tratamiento de la estenosis uretral en un centro de referencia de México}

Ruiz-Hernández A, ${ }^{1}$ Campos-Salcedo JG, ${ }^{2}$ López-Silvestre JC, ${ }^{3}$ EstradaCarrasco CE, ${ }^{3}$ Mendoza-Álvarez LA, ${ }^{3}$ y colaboradores.

Resumen

ANTECEDENTES: la estenosis uretral es una alteración provocada por una cicatriz que disminuye el lumen de la uretra. Hasta el momento existe información limitada del tratamiento y evolución de la enfermedad en la población mexicana.

OBJETIVO: evaluar el origen, tratamiento quirúrgico y evolución de pacientes con estenosis uretral en un centro de concentración mexicano.

MATERIALES Y MÉTODOS: estudio retrospectivo, del que se obtuvieron los datos de pacientes atendidos en el Hospital Central Militar con diagnóstico de estenosis uretral, intervenidos quirúrgicamente entre febrero de 2012 y junio de 2016.

RESULTADOS: se identificaron 174 pacientes intervenidos de estenosis uretral con edad promedio de 58.2 años. El 94.2\% (164) de los casos se intervino por estenosis de uretra anterior y $5.8 \%$ (10) posterior. La uretrotomía interna fue la cirugía más frecuente en $70.1 \%$ (115; 78 con láser y 37 con corte en frío) de los pacientes con estenosis de uretra anterior, con tasas de éxito de $68.5 \%$ en el primer procedimiento y de $81.3 \%$ en uretroplastia con injerto de mucosa oral. La uretroplastia término-terminal fue el principal procedimiento en $60 \%$ de los pacientes con uretra posterior, con tasa de éxito de $61.5 \%$. En total se realizaron 41 uretroplastias (35 uretra anterior, 6 uretra posterior), con tasa de éxito de $73.8 \%$.

CONCLUSIONES: las tasas de éxito de la uretrotomía interna y la uretroplastia son similares a las obtenidas en los centros de referencia mundial.

PALABRAS CLAVE: estenosis uretral; uretroplastia; uretrotomía interna.

Rev Mex Urol. 2017 Jul-Aug;77(4):265-271.

\section{Origin, progression, and treatment of urethral stricture at a Mexican referral center}

Ruiz-Hernández A, ${ }^{1}$ Campos-Salcedo JG, ${ }^{2}$ López-Silvestre JC, ${ }^{3}$ EstradaCarrasco CE, ${ }^{3}$ Mendoza-Álvarez LA, ${ }^{3}$ et al.

\section{Abstract}

BACKGROUND: Urethral stricture is an alteration caused by a scar that reduces the urethral lumen. At present, there is little information

\author{
${ }^{1}$ Residente de Urología. \\ 2 Jefe del servicio de Urología. \\ ${ }^{3}$ Adscrito al servicio de Urología. \\ Hospital Central Militar, Ciudad de México, México. \\ Recibido: diciembre 2016 \\ Aceptado: julio 2017 \\ Correspondencia \\ Dr. José Alfredo Ruíz Hernández \\ alfruiz_3@hotmail.com
}

Este artículo debe citarse como

Ruiz-Hernández A, Campos-Salcedo JG, LópezSilvestre JC, y col. Origen, evolución y tratamiento de la estenosis uretral en un centro de referencia de México. Rev Mex Urol. 2017 jul-agos;77(4):265-271. DOI: https://doi.org/10.24245/revmexurol.v77i4.1114 
on the management and progression of this pathology in the Mexican population.

OBJECTIVE: To evaluate the origin, surgical treatment, and progression of urethral stricture at a Mexican referral center.

MATERIALS AND METHODS: A retrospective study was conducted utilizing the data of patients treated at the Hospital Central Militar that were diagnosed with urethral stricture and surgically managed within the time frame of February 2012 and June 2016.

RESULTS: One hundred seventy-four patients underwent surgical treatment for urethral stricture during the study period. The mean age of the patients was 58.29 years. A total of $94.2 \%$ (164) of the patients had anterior urethral stricture and 5.8\% (10) had strictures of the posterior urethra. Internal urethrotomy was the most common procedure in anterior urethral stricture, with $70.1 \%$ cases $(115 ; 78$ with laser and 37 with cold knife). The first procedure had a success rate of $68.5 \%$ and urethroplasty with a buccal mucosa graft achieved a success rate of $81.3 \%$. The main procedure in the patients with posterior urethra was end-to-end urethroplasty, with $60 \%$ of cases, and a success rate of $61.5 \%$. In total, 41 urethroplasties were performed (35 in the anterior urethra and 6 in the posterior urethra), achieving a success rate of $73.8 \%$.

CONCLUSIONS: The success rates of internal urethrotomy and urethroplasty at our center were similar to those at referral centers, worldwide.

KEYWORDS: Urethral stricture; Urethroplasty; Internal urethrotomy

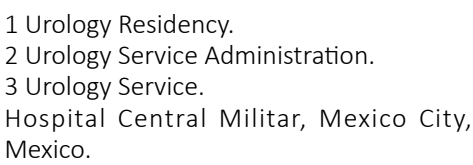

Correspondence

Dr. José Alfredo Ruíz Hernández

alfruiz_3@hotmail.com

\section{ANTECEDENTES}

La estenosis uretral es una alteración con tasa de incidencia de 200 a 1200 casos, con incremento a partir de los 50 años de edad. ${ }^{1}$ La estenosis uretral repercute negativamente en la calidad de vida de los pacientes y eleva los costos de salud, pues se estima que cada año se invierten hasta 190 millones de dólares en el tratamiento de esta enfermedad. ${ }^{1}$ Aunque la uretroplastia se considera el tratamiento de elección para la estenosis de uretra, se han llegado a reportar entre 8.3 y $18.7 \%$ de recurrencias en los centros de mayor experiencia. ${ }^{2}$ Entre las principales causas de estenosis uretral se incluyen: liquen escleroso, traumatismo, iatrogenia e infecciones; ${ }^{3}$ sin embargo, las dos primeras se asocian con mayor riesgo de recurrencia y complicaciones posquirúrgicas. ${ }^{3}$ A pesar de ser una enfermedad con gran repercusión en la calidad de vida, el seguimiento para identificar las complicaciones e implementar el tratamiento de la recurrencia aún no se encuentra estandarizado a nivel mundial. Algunos estudios señalan que $50 \%$ de las recurrencias ocurren en el primer y segundo año posteriores a la uretroplastia; ${ }^{4}$ sin embargo, se ha reportado una tasa de $29 \%$ en los siguientes 5 años del procedimiento quirúrgico.

El propósito de este estudio es identificar el origen, evolución y tratamiento de la estenosis uretral en un centro de referencia de México, 
pues hasta el momento no existe una base de datos concretos del comportamiento de esta enfermedad en la población mexicana.

\section{MATERIALES Y MÉTODOS}

Estudio retrospectivo efectuado mediante el análisis de los expedientes electrónicos de pacientes intervenidos quirúrgicamente por algún tipo de estenosis de uretra, entre febrero de 2012 y junio de 2016, en el Hospital Central Militar. Los pacientes se clasificaron según el sitio de estenosis en: uretra anterior (meato uretral, fosa navicular, uretra peneana y uretra bulbar) y uretra posterior (membranosa y prostática).

\section{RESULTADOS}

Se localizaron 200 expedientes de pacientes intervenidos de estenosis uretral; sin embargo, se excluyeron 26 casos debido a que solamente recibieron tratamiento con calibración, mediante el paso del cistoscopio durante la revisión inicial. Los 174 pacientes restantes tuvieron edad promedio de 58.2 años (39-83 años). Las características generales se muestran en el Cuadro 1. Se registraron síntomas obstructivos en $61.4 \%(n=107)$, irritativos en $12.6 \%$ ( $n=$ 22) y mixtos en $30.5 \%(n=44)$ de los casos. Todos los pacientes tuvieron infección de vías urinarias antes de establecer el diagnóstico.

El Cuadro 2 muestra el tratamiento quirúrgico de los pacientes según el sitio de la estenosis uretral.

En $94.2 \%(n=164)$ de los casos se reportó estenosis en la uretra anterior, de los cuales $41.5 \%$ ( $n=$ 69) requirió tratamiento inicial con sonda de cistostomía. Las causas más frecuentes de estenosis anterior fueron: colocación de sondas transuretrales en $46.3 \%(n=76)$, cirugía endourológica transuretral en $32.3 \%(\mathrm{n}=53)$, prostatectomía radical en $14.6 \%(n=24)$, traumatismo en $6.1 \%$ ( $n=10)$ e infección de transmisión sexual en
Cuadro 1. Características epidemiológicas de los pacientes

\begin{tabular}{|c|c|}
\hline Edad (años) & 58.2 (límites 39-83) \\
\hline Seguimiento (meses) & 17 (límites 3-32) \\
\hline $\begin{array}{l}\text { Tabaquismo } \\
\text { Sí } \\
\text { No }\end{array}$ & $\begin{array}{l}\mathrm{n}=94(54.3 \%) \\
\mathrm{n}=80(45.7 \%)\end{array}$ \\
\hline $\begin{array}{l}\text { IMC } \\
\qquad 25 \\
<25\end{array}$ & $\begin{array}{l}\mathrm{n}=99(57.2 \%) \\
\mathrm{n}=75(42.8 \%)\end{array}$ \\
\hline $\begin{array}{l}\text { Comorbilidades } \\
\text { Diabetes mellitus } \\
\text { Hipertensión arterial sistémica } \\
\text { Ambas }\end{array}$ & $\begin{array}{l}\mathrm{n}=38(21.8 \%) \\
\mathrm{n}=3520.1 \%) \\
\mathrm{n}=21(12.1 \%)\end{array}$ \\
\hline Radioterapia & $\mathrm{n}=3(1.7 \%)$ \\
\hline Disfunción eréctil & $\mathrm{n}=99(56.3 \%)$ \\
\hline $\begin{array}{l}\text { Incontinencia } \\
\text { Esfuerzo } \\
\text { Urgencia } \\
\text { Mixta }\end{array}$ & $\begin{array}{c}\mathrm{n}=31(17.8 \%) \\
\mathrm{n}=32(18.3 \%) \\
\mathrm{n}=6(3.4 \%)\end{array}$ \\
\hline
\end{tabular}

Cuadro 2. Intervención quirúrgica según el sitio de la estenosis

\begin{tabular}{|c|c|c|}
\hline Tratamiento & $\begin{array}{c}\text { Uretra } \\
\text { anterior } \\
\text { n (\%) }\end{array}$ & $\begin{array}{l}\text { Uretra } \\
\text { posterior } \\
\text { n (\%) }\end{array}$ \\
\hline $\begin{array}{l}\text { Uretrotomía interna } \\
\text { Láser holmio } \\
\text { Corte en frío }\end{array}$ & $\begin{array}{c}115(70 \%) \\
78 \\
37\end{array}$ & $\begin{array}{c}4(40 \%) \\
4\end{array}$ \\
\hline $\begin{array}{l}\text { Uretroplastia } \\
\text { Injerto de mucosa oral } \\
\text { Colgajo de piel del prepucio } \\
\text { Colgajo de piel escrotal } \\
\text { Término-terminal }\end{array}$ & $\begin{array}{c}35(21.4 \%) \\
32 \\
2 \\
1\end{array}$ & $6(60 \%)$ \\
\hline Perineostomía & $13(7.9 \%)$ & \\
\hline Meatoplastia & $1(0.7 \%)$ & \\
\hline Total & 164 & 10 \\
\hline
\end{tabular}

$0.6 \%(n=1)$. Para establecer el diagnóstico se realizaron: uretrografía retrógrada en 94 pacientes y cistografía miccional en 36 (en 34 casos se identificó durante el procedimiento de cistoscopia). Se registró una longitud media de estenosis anterior de $2.8 \mathrm{~cm}(0.5$ a 8$)$. El $70.1 \%(n=115)$ de los pacientes se intervino de uretrotomía interna (78 con láser holmio y 37 con corte en frío), 19.5\% ( = 32) de uretroplastia con colocación de injerto 
de mucosa oral, $7.9 \%(n=13)$ de perineostomía, $1.3 \%(n=2)$ de uretroplastia con colgajo de piel de prepucio y $0.6 \%(n=1)$ de meatoplastia y uretroplastia con injerto de piel escrotal. De los pacientes con uretrotomía interna, 31.5\% $(\mathrm{n}=$ 36: 20 con corte en frío y 16 con láser holmio) requirió intervención quirúrgica adicional, logrando solo en 14 casos permeabilidad uretral, mientras que los 22 restantes se sometieron a otro procedimiento más, es decir, uretrotomía por tercera ocasión, colocación de sonda transuretral o procedimiento abierto. De los procedimientos abiertos, $53.1 \%$ de los pacientes (18 uretroplastias con injerto de mucosa oral, 4 perineostomías, 2 uretroplastias con colgajo de piel de prepucio, 1 uretroplastia con colgajo de piel escrotal y 1 meatoplastia) tuvo complicaciones (Cuadro 3).

En 5.8\% $(\mathrm{n}=10)$ de los casos se diagnosticó estenosis de uretra posterior, con una longitud media de $1 \mathrm{~cm}(0.5$ a 1$)$, quienes requirieron cistostomía como tratamiento inicial. Las principales causas de estenosis posterior fueron: procedimientos endourológicos transuretrales $60 \%(n=6)$, accidentes automovilísticos $30 \%$ $(\mathrm{n}=3)$ y atropellamiento $10 \%(\mathrm{n}=1)$. En $40 \%$ $(n=4)$ de los pacientes se practicó uretrotomía interna con láser holmio y $60 \%(n=6)$ uretroplastia término-terminal. De los pacientes con uretrotomía interna, solo 1 logró permeabilidad uretral, 2 continuaron con cistostomía y otros dos se sometieron a procedimiento abierto. El Cuadro 4 muestra el tratamiento con uretroplastia término-terminal y las complicaciones.

Como método diagnóstico de las recurrencias o complicaciones posquirúrgicas, $100 \%(\mathrm{n}=$ 60) requirió uretrografía retrógrada, $64.7 \%$ (n = 39) cistoscopia y $21.5 \%(n=13)$ uretrografía retrógrada y cistografía miccional.

\section{DISCUSIÓN}

La información disponible acerca de la epidemiología, tratamiento y seguimiento de pacientes con estenosis de uretra en México es limitada, con pocos artículos sistemáticos o retrospectivos y cantidad de pacientes muy baja. ${ }^{6,7}$ Como método diagnóstico, la uretrografía retrógrada fue el estudio de elección en la mayoría de nuestros pacientes, pues ha demostrado sensibilidad de $75-100 \%$ y especificidad de $72-97 \% .^{8} \mathrm{Sin}$ embargo, la cistoscopia sigue siendo el estudio de referencia para establecer el diagnóstico de estenosis uretral, con una sensibilidad de 95\% y especificidad de $93 \% .^{8}$ En nuestro estudio la iatrogenia fue la principal causa de estenosis uretral, sobre todo la colocación de sondas transuretrales en $43.1 \%$ de los pacientes. Liu y sus colaboradores reportaron esta misma variable como causa de estenosis uretral (33\%), seguida de traumatismo (15\%) y resección transuretral de próstata $(9 \%){ }^{8}$

En pacientes con estenosis de uretra anterior de $2-4 \mathrm{~cm}$, la uretrotomía interna sigue siendo el tratamiento de primera línea. ${ }^{10}$ En nuestro estudio se logró $68.6 \%$ de éxito en la primer uretrotomía interna de las estenosis en uretra anterior, similar a lo reportado por Buckley y su grupo, quienes señalaron una tasa de éxito de $50-70 \%$ en pacientes con la misma longitud de estenosis. ${ }^{9}$ De igual forma, en los casos con recurrencia posterior a la uretrotomía interna, al realizar el segundo procedimiento se logró una tasa de éxito de $9-53 \%$, hasta llegar a 100\% en quienes requirieron el tercer procedimiento. ${ }^{9-10}$ En pacientes con estenosis de uretra posterior, la primer uretrotomía fue exitosa en $75 \%$, similar a lo reportado en diversos centros de referencia internacionales, en donde se han registrado tasas de éxito de 71 y $47 \%$ en la primera y segunda uretrotomías, respectivamente. ${ }^{11}$ De acuerdo con múltiples estudios comparativos, ${ }^{11}$ durante la uretrotomía interna el uso de láser no es más efectivo que el corte en frío.

La uretroplastia es el tratamiento de elección en pacientes con estenosis uretrales anteriores y posteriores. Barbagli y sus coautores ${ }^{12}$ señalan 

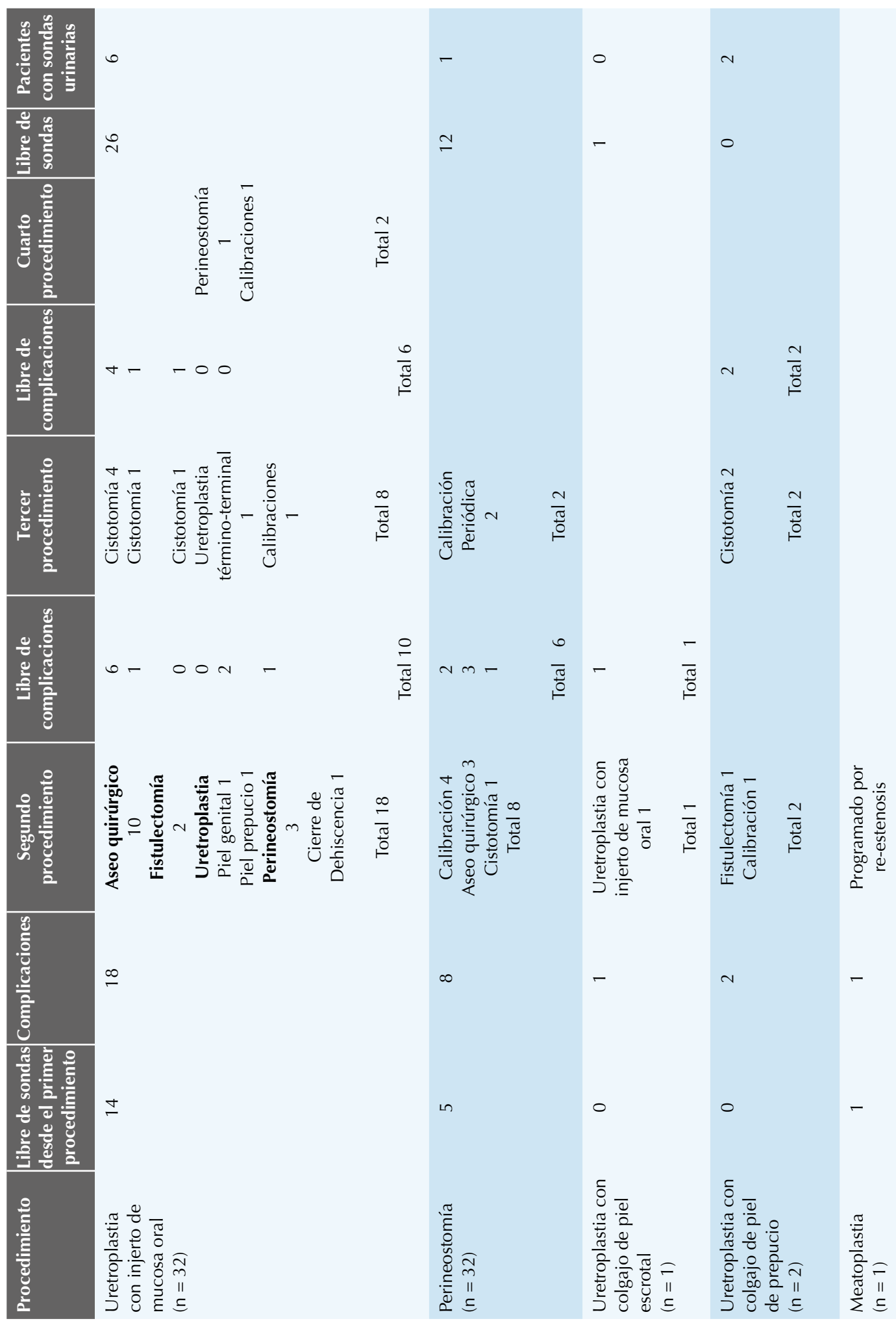


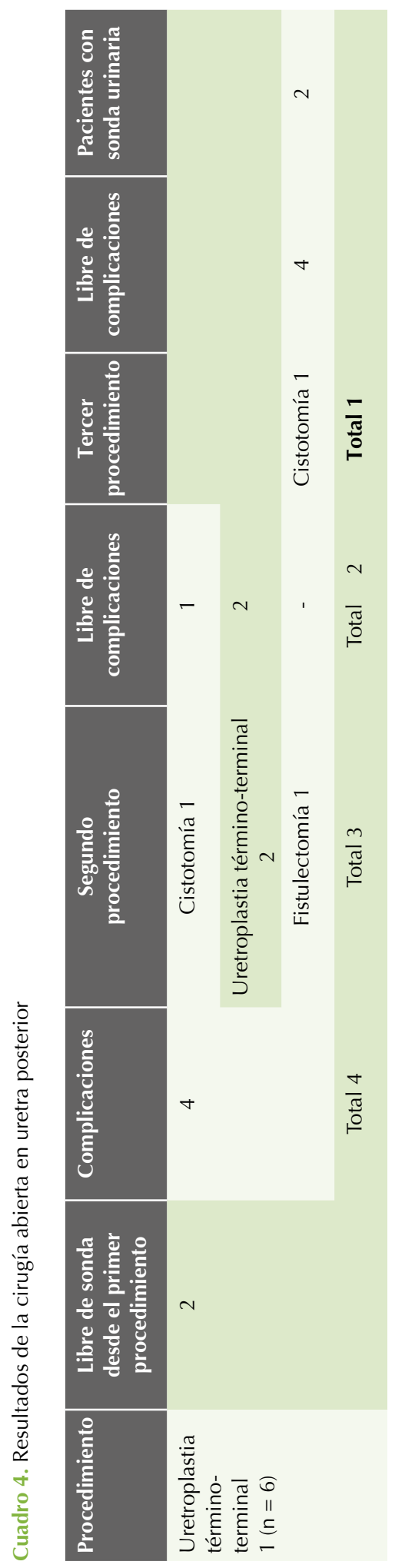

una tasa de éxito entre 91 y $98 \%$ en el tratamiento de estenosis de uretra anterior menor a $3 \mathrm{~cm}$ con la uretroplastia término-terminal y $65.8 \%$ en estenosis mayores de $3 \mathrm{~cm}$ con la uretroplastia de aumento. Sin embargo, Andrich y sus colaboradores reportaron recurrencia de $42 \%$ en la uretroplastia de aumento y $14 \%$ en la término-terminal, con seguimiento a 15 años. ${ }^{13} \mathrm{~A}$ la mayoría de los pacientes que se interviene de uretroplastia de aumento se les colocan colgajos de mucosa oral, pues son fáciles de obtener, resistentes, permiten colgajos de adecuado grosor, son de fácil colocación, y tienen epitelio grueso y lámina vascular; por tanto, los convierte en el tejido de elección. ${ }^{14}$ Para el tratamiento de la estenosis de uretra posterior es necesario evaluar el origen de la alteración, con la finalidad de establecer el tratamiento adecuado. De acuerdo con la Asociación Americana de Urología (AUA), el primer paso consiste en la realineación del defecto uretral, con colocación de una sonda transuretral, y diferir a uretroplastia para un segundo tiempo. Según el paciente y su evolución, la reconstrucción uretral puede retrasarse de 6 meses a 4 años. ${ }^{10}$ En estos casos se han reportado tasas de éxito de $90-98 \%$ cuando se realiza uretroplastia término-terminal en estenosis menores a $3 \mathrm{~cm}$, mientras que en estenosis mayores la uretroplastia de aumento es el tratamiento de elección, con tasas de éxito estimadas en $64 \% .^{11}$ El metanálisis de $S_{\text {mith }}{ }^{15}$ reportó 21 a $70 \%$ de éxito en el tratamiento de la estenosis de uretra posterior por causas iatrogénicas al realizar uretroplastia de aumento.

\section{CONCLUSIONES}

En nuestro centro se definió como éxito el no requerimiento de colocación de sondas urinarias. Con ese parámetro se evidenció $43.7 \%$ de falla en las uretroplastias con injerto de mucosa en el primer tiempo quirúrgico, logrando disminuirlo a $18.7 \%$ en el segundo, tercer o cuarto procedimiento. Se logró una tasa de éxito de $61.5 \%$ durante el primer tiempo de la coloca- 
ción de perineostomías, incluso aumentó a 92\% después del segundo o tercer procedimiento. Con estos resultados se demuestran tasas de éxito positivas en la práctica de procedimientos abiertos para el tratamiento de las estenosis de uretra; sin embargo, es necesario ampliar la casuística para lograr porcentajes de éxito en el primer tiempo quirúrgico similares a los de los centros de referencia mundiales. Algunos estudios señalan cifras similares en cuanto a origen, diagnóstico y tratamiento de estenosis uretral a la de nuestro centro; por tanto, el comportamiento de la población mexicana no es diferente al de otros países. Para confirmar esta aseveración se requieren estudios interinstitucionales con mayor número de pacientes.

No se recibió financiamiento para la elaboración de este trabajo.

Ninguno de los autores manifestó tener algún conflicto de interés.

\section{REFERENCIAS}

1. Santucci RA, Joyce GF, Wise M. Male urethral stricture disease. J Urol. 2007;177:1667-1674.

2. Meeks JJ, Erickson BA, Granieri MA, Gonzalez CM. Stricture recurrence after urethroplasty: a systematic review. J Urol. 2009;182:1266-1270.

3. Stein DM, Thum DJ, Barbagli G, et al. A geographic analysis of male urethral stricture aetiology and location. BJU Int. 2013;112:830-834.
4. Breyer BN, McAninch JW, Whitson JM, et al. Multivariate analysis of risk factors for long-term urethroplasty outcome. J Urol. 2010;183:613-617.

5. Blaschko SD, McAninch JW, Myers JB, Schlomer BJ, Breyer BN. Repeat urethroplasty after failed urethral reconstruction: outcome analysis of 130 patients. J Urol. 2012;188:2260-2264.

6. Cisneros-Chávez R, Aragón-Castro MA, Morales-Ordaz O. Estenosis uretral: etiología y tratamiento. Experiencia en el Centro Médico ISSEMYM. Rev Mex Urol 2013;73(5):229-236.

7. Serrano-Brambila EA, Moreno-Alcázar OM, Neri-Páez E, Sánchez-Martínez LC, Hernández-Ordóñez OF. Guía de Práctica Clínica. La estenosis de uretra postraumática en el hombre. Rev Med Inst Mex Seg Soc 2013;51(4):472-479.

8. Liu JS, Dong C, Gonzalez CM. Risk factors and timing of early stricture recurrence after urethroplasty. Urology. 2016 Sep;95:202-7

9. Buckley JC, Heyns C, Gilling P, Carney J. SIU/ICUD Consultation on Urethral Strictures: Dilation, internal urethrotomy, and stenting of male anterior urethral strictures. Urology 2014;83(3 Suppl):S18-22.

10. Wessells H, Angermeier WK, Elliott PS, Gonzalez MC, Kodama TR, et al. Male urethral stricture. American Urological Association Guidelines. 2016.

11. Jordan G, Chapple C, Heyns C. Urethral strictures. An international consultation on urethral strictures. American Urological Association, October, 2010.

12. Barbagli G, Guazzoni G, Lazzeri M. One-stage bulbar urethroplasty: Retrospective analysis of the results in $\mathbf{3 7 5}$ patients. Eur Urol. 2008;53(4):828-833.

13. Eltahawy EA, Virasoro R, Schlossberg SM, et al. Long-term follow-up for excision and primary anastomosis for anterior urethral strictures. J Urol. 2007;177(5):1803-1806.

14. Bürger RA, Müller SC, el-Damanhoury $H$, et al. The buccal mucosal graft for urethral reconstruction: A preliminary report. J Urol. 1992;147(3):662-664.

15. Smith TG III. Current management of urethral stricture disease. Indian J Urol. 2016;32(1):27-33. 